\title{
Onlinebeteiligung als politische Bildung
}

\section{Robert Behrendt}

Partizipation ist in Deutschland seit den 1990er-Jahren wieder ein relevantes Thema in der Kinder- und Jugendhilfe und politischen Bildungsarbeit und sogar in öffentlichen Verwaltungen. Sie ist seitdem auch zu einem nicht unbedeutenden (und politisch gewollten) Gradmesser des Zustandes der demokratischen Kultur in der Bundesrepublik geworden. Mitbestimmung als Fundament von Partizipation war in institutionalisierter Form zwar von Beginn an integraler Bestandteil demokratischer Gesellschaften und Organisationen, in nichtverfassten oder unkonventionellen Formen aber immer auch Gegenstand demokratischer Kontroversen. Ob als wilde Streiks, Sit-ins, Besetzungen, Blockaden oder Festivals, als Happening, Bürgerinitiative oder soziale Bewegung - die Äußerungsformen eines zivilgesellschaftlichen Mitbestimmungs- und Gestaltungswillen sind vielgestaltig, kreativ und bisweilen provokativ.

Durch das frühe Freiheitsversprechen des Web 1.0 („Im Internet weiß niemand, dass du ein Hund bist.“ Steiner 1993) bekamen Beteiligungsanliegen zusätzliche Impulse und die Erwartungen an den neuen digitalen öffentlichen Raum nahmen teils messianische Züge an. Mit Entwicklung der sozialen Medien wurde dieses naive Heilsversprechen nicht nur technokratisch bestärkt, sondern zusätzlich in kommerzielle Interessen umgemünzt. In 2020 ist diese Naivität teils einer Resignation gewichen. Grund genug, sich als politischer Bildner weder der einen noch der anderen Seite zuzuschlagen.

Nichtverfasste und unkonventionelle Formen von Partizipation haben sich durch neue Technologien und die seit den 2000er-Jahren einsetzende Digitalisierung des Alltags wortwörtlich eine neue Dimension erschlossen. Vermehrte

R. Behrendt $(\varangle)$

mediale pfade, Berlin, Deutschland

E-Mail: robert.behrendt@medialepfade.org 
Zugangsmöglichkeiten zum Internet, smarte mobile Endgeräte für Nutzende sowie kommerzielle Plattformen, die infrastrukturell das Social Web gestalteten, erlauben vielen Menschen einfacheren Zugang zu Information, erweiterte Möglichkeiten zu Austausch und Kommunikation und damit neue Formen der Organisation. Social Media über Dienste wie YouTube oder Instagram/Facebook und Messengerdienste wie WhatsApp, Telegram oder Signal haben die politische Kommunikation, die Kommunikation über Politik und politische Fragestellungen und damit auch die gesellschaftlichen Diskurse beschleunigt, polarisiert und radikalisiert.

Die Kommunikationsräume, die kommerzielle Plattformen und technische Dienste bieten, sind natürlich auch fester Bestandteil jugendlicher Lebenswelten geworden. In Deutschland liegt laut JIM-Studie 2018 die Ausstattungsrate mit Smartphones bei den 12- bis 19-Jährigen bei $97 \%$, womit nahezu alle Jugendlichen ein Gerät besitzen. Diese weite Verbreitung eines fast universellen Gerätes zur Nutzung und Produktion digitaler Medien bietet beachtliches Potenzial für Bildungsprozesse und kann jungen Menschen aller Milieus Möglichkeiten zu gesellschaftlicher Teilhabe bieten.

Sowohl bei den liebsten Internetangeboten als auch bei wichtigsten Apps 2018 liegen die Angebote von YouTube, Instagram und WhatsApp auf den ersten drei Plätzen (Feierabend et al. 2018, S. 35-36). Zum einen zeigt sich an der Wahl dieser technischen Dienste die Bandbreite der von ihnen rezipierten oder produzierten Medien. Webvideos, Musik, Sprachnachrichten, Fotos, Texte und Symbole können distribuiert werden und werden von Jugendlichen tatsächlich genutzt. Zum anderen legen die von Jugendlichen verwendeten Dienste eine Lesart ihres Nutzungsverhalten nahe, wonach sich ihre Weisen der Kommunikation, Information und Unterhaltungen einander durchdringen und eine Unterscheidung von online und offline zunehmend obsolet erscheinen lassen. Etwas, das Luciano Floridis in seinem Werk Die 4. Revolution als „Onlife“ (2015, S. 87-89) beschreibt, ist für junge Menschen bereits alltäglich geworden. Sie kommunizieren mittels sozialer Medien über Inhalte in den sozialen Medien, die sich wiederum aus ihrem Mediennutzungsverhalten und erlebten Alltag speisen, darauf zurückwirken und diesen überformen.

Diese gesellschaftlichen Entwicklungen hin zu medial vermittelter Kommunikation bestärken Ansätze in der politischen Bildung, die Konzepte zum Medienkompetenzerwerb und der aktiven Medienarbeit nutzen. Urteilskraft und Mündigkeit vor dem Hintergrund zeitgenössischer technologischer Entwicklungen auszubilden, bedeutet das Technische vor allem politisch zu denken und als Bündel gesellschaftlicher Fragen zu behandeln, auf die es keine vorgefertigten 
Antworten geben kann. Diese Fragen müssen beständig neu gestellt und Antworten darauf zudem ausgehandelt werden. Unter dieser Perspektive möchte ich einen detaillierten Blick auf zwei medienpädagogische und politisch-bildnerische Projekte werfen, die sich dezidiert mit Fragestellungen beschäftigt haben, die seit etwa 2015 durch die sozialen Medien virulent geworden sind. Das erste Projekt, „\#NichtEgal“, befasste sich mit dem Umgang mit Hate Speech im Internet und „How2Influence“, das zweite Projekt, mit der Rolle von Influencing im Social Web.

Beide Projekte glichen sich in ihrem grundlegenden Ansatz und arbeiteten mit derselben Zielgruppe. Gemeinsam mit 14- bis 16-Jährigen wurden aktuelle gesellschaftliche Fragestellungen bearbeitet, indem die Jugendlichen die eigene Technik nutzten, um Medienprodukte zu erstellen, die Perspektiven auf die Phänomene „Hate Speech“ bzw. „Influencing“ entwerfen und Antwortmöglichkeiten entwickeln. Die erarbeiteten Perspektiven und Antworten verblieben jedoch nicht im geschützten, nichtöffentlichen Setting einer Präsenzveranstaltung, sondern sie wurden über die sozialen Medien publiziert und öffentlich zur Debatte gestellt. D. h. die Auseinandersetzung mit den Themen „Hate Speech“ bzw. „Influencing“ wurde zu Teilen in eben demjenigen Medium ausgetragen, das diese Phänomene überhaupt erst möglich gemacht hatte und in dieser Form entstehen ließ.

Durch diesen medial vermittelten Zugang zum Thema wurden zwei zusätzliche Ebenen in beide Projekte eingebracht. Zum einen machte die mediale Aufbereitung und Arbeit in den sozialen Medien eine Auseinandersetzung mit den Regeln und Funktionsweisen des Mediums notwendig, schließlich sollten die Themen „Hate Speech“ bzw. „Influencing“ vor dem Hintergrund dieser Regeln verhandelt werden können. Zum anderen ermöglichte der Einsatz von Instagram oder YouTube es den Jugendlichen, ihre Perspektiven auf diese aktuellen gesellschaftlichen Fragen in die öffentlichen Debatten einzubringen, d. h. beide Projekte eröffneten ihnen Mittel und Wege der gesellschaftlichen Teilhabe und sozialen Beteiligung. Onlinebeteiligung war auf diese Weise sowohl Mittel als auch Zweck der Projektentwürfe und konnte den Jugendlichen ihre eigene Agency und Selbstwirksamkeit vor Augen führen. Nicht zuletzt wurde durch diese Projektentwürfe die Rolle der Jugendlichen in digitalen ,öffentlichen“ Räumen wie Social-Media-Plattformen thematisiert und die zentrale Frage nach der eigenen Verantwortung gestellt. 


\section{1 \#NichtEgal}

In der Betrachtung von \#NichtEgal ${ }^{1}$ möchte ich mich auf den medienpädagogischen Projektteil beschränken und nicht die Initiative als Ganzes beleuchten, auch wenn es hier so interessante wie kontroverse Ansatzpunkte gäbe, um die Rolle eines globalen Akteurs der Technologiebranche als Policy Maker im Bildungsbereich zu verhandeln. \#NichtEgal wurde als YouTube-Initiative durch Google Germany verantwortet und startete bereits 2016 mit einer Kampagne, die schnell viel Widerspruch erfuhr. Die Kritik entlud sich vor allem daran, dass die Initiative, die sich für mehr "Respekt und Toleranz im Netz" und gegen Hate Speech einsetzten wollte, im ersten Kampagnenvideo teils mit Creatorn kooperierte, die selbst nur als schlechte Beispiele für das Thema herhalten konnten. YouTuberinnen und YouTuber wie Chris Manazidis, Hand of Blood oder Dagi Bee waren in ihren eigenen Posts nicht gerade durch diskriminierungsfreie Sprache aufgefallen. Der Blogger Christian Brandes (2016) hatte in einem Video auf diese Schieflage im Rahmen einer Initiative zur Wertevermittlung hingewiesen und damit ein Netzdebatte losgetreten.

Vor diesem Hintergrund war es eine besondere Herausforderung, die Initiative und das Thema pädagogisch in die Fläche zu tragen. Es war schließlich die durch Google Germany garantierte Unabhängigkeit der beteiligten medienpädagogischen Einrichtungen bei der Konzeption und Durchführungen des Projekts, die es diesen zehn Partnerorganisationen ermöglichte, ein mehrstufiges Workshopprogramm für Schulen umzusetzen und deutschlandweit auszurollen. Über sechzig Schulen und 5000 Schülerinnen und Schüler wurden erreicht in nahezu allen Bundesländern. Dank der frühen Einbindung der medienpädagogischen Partner in der Konzeption und bei der Erstellung der Bildungsmaterialien sowie durch die umfangreiche Vorbereitung der Trainerinnen, Trainer und Lehrkräfte war es möglich, das Workshopkonzept zu skalieren und auf einem vergleichsweise hohen Standard in sehr unterschiedlichen Regionen der Bundesrepublik durchzuführen.

Die Gesamtkonzeption der Workshops war als Peer-Education und darum auf Mehrstufigkeit angelegt. Nach der Ausschreibung des Programms und der Bewerbung der Schulen auf eine Teilnahme wurden in enger Abstimmung mit den regionalen Partnern die Lehrkräfte inhaltlich und organisatorisch vorbereitet. Der erste Workshopeinsatz in den Schulen war als „Mentor*innen-Training“, der zweite Workshopeinsatz zwei bis vier Wochen später als „Aktionstag“ konzipiert. Im „Mentor*innen-Training“ durchliefen Schülerinnen und Schüler der 9. oder 10. Klassen eine eintägige Vorbereitung, um inhaltlich selbständig mit

\footnotetext{
${ }^{1}$ Siehe die Projektwebseite für weitere Informationen: https://nichtegal.withyoutube.com.
} 
Schülerinnen und Schülern der 7. und 8. Klassen den ebenfalls eintägigen „Aktionstag“ durchführen zu können. Sie absolvierten bereits im vorbereitenden „Mentor*innen-Training“ alle Phasen des „Aktionstages“ selbst und reflektierten zudem ihre Rolle als Lehrende vor einer jüngeren Klasse. Immer drei bis fünf Schülerinnen und Schüler der 9. oder 10. Klassen übernahmen am „Aktionstag“ schließlich eine 7. oder 8. Klasse. Medienpädagoginnen und Medienpädagogen, politische Bildnerinnen und Bildner und die Lehrkräfte standen dabei vor allem unterstützend zur Seite, um organisatorische Fragen zu klären oder bei Bedarf und auf Wunsch die „Mentor*innen“ inhaltliche Ratschläge zu geben.

Kern des „Mentor*innen-Trainings“ und des „Aktionstages“ war die themenzentrierte Produkterstellung. Die Schülerinnen und Schüler hatten insgesamt sieben Unterrichtsstunden Zeit, um als Gruppen von drei bis sechs Jugendlichen ein etwa einminütiges Video zu erstellen, das ihren Vorschlag für ein tolerantes und respektvolles Miteinander im Netz vermitteln können sollte. Dabei war der gesamte Erstellungsprozess von der Ideenfindung über das Skripten bis hin zu Dreh und Postproduktion klar getaktet. Der Großteil der Zeit, etwa vier Unterrichtsstunden, floss in die inhaltliche Auseinandersetzung, Ideenentwicklung und -ausarbeitung. Die Schülerinnen und Schüler nutzten für den Dreh ihre eigenen Smartphones und waren angehalten den Produktionsprozess so schlank wie möglich zu halten. Die Ergebnisse zeigten sowohl in Formatwahl als auch hinsichtlich ihrer Qualität eine große Spannweite, schließlich wurden alle Entscheidungen durch die Jugendlichen in den Arbeitsgruppen getroffen. Die Jugendlichen nahmen oft das Thema (Cyber-)Mobbing zum Ausgangspunkt für ihre Skripte, genauso bildeten aber auch eigene Diskriminierungserfahrungen im Schulkontext häufiger die inhaltliche Grundlage für die Videos.

Im Verlauf des „Mentor*innen-Trainings“ und des „Aktionstages“ wurde mit allen Teilnehmenden ausführlich die Frage der Veröffentlichung thematisiert, sowohl was die rechtlichen Rahmenbedingungen (Urheberrecht und Persönlichkeitsrecht) betrifft, als auch die Fragen der persönlichen Verantwortung und Exponiertheit als Person in der Öffentlichkeit der sozialen Medien. Alle Arbeitsgruppen konnten sich frei entscheiden, ob sie eine Veröffentlichung wünschten, und diese Entscheidung musste einstimmig fallen. ${ }^{2}$

Der Großteil von Schülerinnen und Schülern, etwa $90 \%$, nahm allerdings Abstand von einer Veröffentlichung. Für die politische Bildung, die Jugendbeteiligung in den sozialen Medien umsetzen will, sollte diese Beobachtung im Blick

\footnotetext{
${ }^{2}$ Die Playlist der Videos aus den Schülerworkshops zu \#NichtEgal online unter https://www. youtube.com/playlist?list=PLc_F-mEKPA-qbZr5waS33FjuSJOajvBoq.
} 
behalten werden, entscheidet sie doch über das Maß an Öffentlichkeit, das solche Projektansätze tatsächlich erreichen können. Die Jugendlichen bei \#Nichtegal waren jedenfalls zurückhaltend bis vorsichtig. Die Entscheidung für eine Veröffentlichung eigener Medienprodukte oder persönlicher Informationen wie das eigene Bild sollte schließlich eine gut informierte, abgewogene und in der Gruppe abgestimmte Entscheidung sein.

\section{$2 \quad$ How2Influence}

Das Projekt How2Influence ist ebenso wie \#NichtEgal ein medienpraktisches Projekt, das am Übergang von politischer Bildung und Medienpädagogik arbeitet. Über die Methode der aktiven Medienarbeit in und mit sozialen Medien bekamen sechzig Jugendliche aus drei Schulen in Berlin und Brandenburg die Möglichkeit, ihre Themen im öffentlichen digitalen Raum zu platzieren und Fragen rund um das Thema „Influencing“ anlassbezogen zu verhandeln und zu reflektieren. Die Ergebnisse ihrer Social-Media-Kanäle wurden in einem gemeinsamen Wettbewerb bewertet und prämiert. Grundlage für diese Bewertung waren dabei Kriterien, die zu Beginn des Projektes gemeinsam erarbeitet wurden (vgl. Behrendt 2019).

Zentrales Anliegen des Projektes war es, die Rolle von Influencing für die Sozialisation und Entwicklung von Wertevorstellungen bei Jugendlichen mit diesen gemeinsam herauszuarbeiten. Die jungen Menschen sollten einen Orientierungsrahmen gewinnen, der ihnen hilft zu verstehen, unter welchen Bedingungen und mit welchen Motiven Influencerinnen und Influencer in den sozialen Medien agieren. Dabei wurde von den Projektentwickelnden ein weiter Begriff von Influencing zur Grundlage genommen, der sowohl monetär als auch sozial und politisch motiviertes Influencing umfasste. Das partizipative Erarbeiten einer gemeinsamen Begriffsgrundlage in den Schulworkshops half den Schülerinnen und Schüler dabei von Beginn an in die Spur.

Die herausfordernde didaktische Grundlage bildete jedoch der medienpraktische Ansatz. Über die Methode der aktiven Medienarbeit verbunden mit dem BYOD-Ansatz (Bring your own device), d. h. mit eigenen Geräten, vornehmlich Smartphones, sollte das Thema Influencing im Medium selbst bearbeitet werden. Die Jugendlichen agierten in den sozialen Medien mit eigenen Kanälen gewissermaßen als beginnende Influencerinnen und Influencer. Zur Auswahl standen Ihnen die Plattformen Instagram und YouTube, auf denen sie eigens für das Projekt eröffnete Kanäle starteten, auf die sie vollen Zugriff hatten. 
Die Schülerinnen und Schüler arbeiteten in Kleingruppen von zwei bis vier Jugendlichen, in wenigen Ausnahmen auch alleine, und hatten insgesamt drei Monate Zeit, ihre Kanäle zu entwickeln. Dabei wurden sie von Medienpädagoginnen und Medienpädagogen durch insgesamt drei ganztägige Schulworkshops pro Schule und die gemeinsame Auftaktveranstaltung unterstützt. Der Hauptteil der eigentlichen Redaktionsarbeit auf ihren Kanälen erfolgte jedoch eigenständig und selbstorganisiert und wurde durch die Medienpädagoginnen und Medienpädagogen ausschließlich digital über Messengerdienste (WhatsApp oder In-App-Messenger bei Instagram) begleitet.

Dieser Punkt bedeutete mit Sicherheit die größte Herausforderung im Projekt, denn eigenständige Medienproduktionen von Jugendlichen im Schulkontext sollten gut motiviert sein und selbst dann sind engagierte Ergebnisse nur von etwa einem Viertel der Teilnehmenden zu erwarten. Im Fall von How2Influence half sicher der von Beginn an angekündigte Wettbewerb aller Kanäle untereinander, Anreize zu schaffen. Bewertungsgrundlage für den Wettbewerb waren, wie erwähnt, die gemeinsam erarbeiteten und abgestimmten Kriterien. Die Kanäle der Jugendlichen konnten also an ihren eigenen Maßstäben gemessen werden, die Kriterien zeigten, welche Schwerpunkte sie selbst setzten, und es war klar, wie auch die Jury nach drei Monaten ihre Entscheidung gewichten würde.

Dabei war für den gesamten Bildungsprozess durchaus entscheidend, dass von der Ausarbeitung der Kriterien beim Auftakt über die drei Zwischenauswertungen in den Schulworkshops bis hin zu den abschließenden Gruppeninterviews vor der abschließenden Preisverleihung gemeinsame Feedback- und Reflexionsrunden der Jugendlichen die medienpraktische Arbeit begleiteten. Erst durch die Öffnung dieser diskursiven Räume, wurden die Jugendlichen in die Lage versetzt, ihr eigenes Social-Media-Handeln mit dem von Influencerinnen und Influencern in Beziehung zu setzen, die Gemachtheit von Selbstbildern und Authentizität zu erfassen und aktuelle Social-Media-Ereignisse zum Anlass zu nehmen, das Medium in seiner gesellschaftlichen Wirkungsbreite zu beleuchten.

Hier zeigte sich bald, dass es ein didaktischer Gewinn war, sich dem Begriff „Influencing“ zwar von seiner werbenden, monetären Funktion her zu nähern, aber stets auch seine politische Dimension im Blick zu behalten. Ereignisse wie die Demonstrationen von Fridays for Future oder der Anschlag auf zwei Moscheen in Christchurch (Neuseeland) fielen in die Projektphase der Schulworkshops und boten bedeutende Anlässe, mit den Jugendlichen die Wirkung medial vermittelter Inhalte zu diskutieren bzw. auch die propagandistischen und gesellschaftspolitischen Aspekte von Influencing zu bearbeiten. 


\section{$3 \quad$ Fazit}

Beide Projekte fanden im Schulkontext statt und die Bedingungen dieser Lernumgebung hatten bedeutenden Einfluss auf die Projektverläufe. Vor allem, wie motiviert die Jugendlichen selbst Impulse ins Projekt einbrachten und ob sie eigenständig im Social Web aktiv waren, wurde durch den verpflichtenden Kontext Schule maßgeblich mitbestimmt. Auch wenn die Anmeldung zu den Projekten freiwillig geschah, sind Abstimmungsprozesse in Schulen nicht in allen Instanzen partizipativ organisiert. Insoweit wurde die Freiwilligkeit der Teilnehmenden, die im Rahmen von Beteiligungsarbeit eigentlich gefordert ist, eingeschränkt.

Die themenzentrierte und aktive Medienarbeit bildete dazu wiederum ein Gegengewicht. In beiden Projekten wurde die inhaltlichen Schwerpunkte „Hate Speech“ bzw. „Influencing“ als Rahmen genutzt, aber die Jugendlichen hatten gestalterische Freiräume in der Themenfindung. Das bedeutete allerdings, dass etwa im Projekt \#NichtEgal eine toxische Netzkultur kaum auf ihre Bedingungen und Strukturen hin beleuchtet wurde, sondern Reflexionen und Themensuche ihren Ausgang mehrheitlich von den singulären Erfahrungen der Jugendlichen nahmen. Das ist mit Blick auf die Motivation der Teilnehmenden sicher ein Vorteil und gleichzeitig eine entscheidende Bedingung für Partizipation. Bei \#NichtEgal ist dabei folgende thematische Verschiebung interessant: Die Videos der Jugendlichen zu einer respektvollen Kommunikation im Netz behandelten weniger die öffentliche Kommunikation auf Plattformen bzw. das Phänomen Hate Speech, als vielmehr ihre persönlichen Erfahrungen in Messengergruppen.

Auch die Kanäle der Jugendlichen im Projekt How2Influence beschäftigten sich nicht dezidiert und reflexiv mit dem Thema „Influencing“, wie es in der ursprünglichen Konzeption angedacht war. Vielmehr standen auch hier ihre persönlichen Interessen im Fokus. Mit Themen wie Gaming, Sport, Mode, Reisen, Ernährung, Haustiere oder Streetart bearbeiteten sie jedoch Themen, die auch und vor allem von Influencerinnen und Influencern bedient werden. Insoweit war es ohne Umwege möglich, Fragen der Selbstdarstellung, Haltung und Wertevermittlung in den sozialen Medien zu thematisieren und anlassbezogen auf die Fragen der Jugendlichen einzugehen, um einen Raum für Reflexion und politische Bildungsprozesse zu öffnen.

Die vierköpfige Jury von How2Influence - zusammengesetzt aus einer Jugendlichen, einem Medienpädagogen, einer Influencerin und einer Social-MediaAgentin - kam in der Bewertung der 17 Kanäle zu einem klaren Votum. Grund dafür war der deutliche Unterschied im Engagement der Jugendlichen, das sich 
in den Arbeitsergebnissen spiegelte. An hochwertigeren Posts ließ sich die investierte Zeit und die konzeptionelle Ausdauer ablesen, währenddessen die Timelines der Kanäle, die Influencing nebenbei betrieben, eher unfertige Ergebnisse zeigten.

Insoweit scheint auch die Rückmeldung der Teilnehmenden authentisch, dass sie nicht erwartet hatten, wie aufwendig gute Contentproduktion und regelmäßige Kanalveröffentlichungen doch seien. Sie konnten in der eigenen Arbeit die Erfahrung machen, welcher Aufwand auch durch professionelle Influencerinnen und Influencer betrieben werden muss und inwieweit diese medial vermittelten Selbstbilder bestimmten Produktionsregeln unterliegen. Hier zeigte sich ihnen deutlich die Inszenierung und Gemachtheit dieser digitalen Repräsentationen.

Bei beiden Projekten nahm der schulische Kontext entscheidenden Einfluss auf die Form der Beteiligungsarbeit, denn es steht außer Frage, dass Schulpflicht und freiwillige Online-Partizipation in Konflikt stehen. Entsprechend haben wir versucht, für beide Projekte möglichst Projekttage im Schulbetrieb oder fakultativen Unterricht $\mathrm{zu}$ nutzen, um die nötigen Freiräume $\mathrm{zu}$ schaffen. Außerdem sollte auf jede Arte von Benotung und andere Disziplinarmaßnahmen verzichtet werden. Freie Themenwahl, die Nutzung eigener Geräte und volle Kontrolle über die Kanäle sollte zusätzliche Motivation schaffen, damit die Projekte aus der Schule in die Freizeit hineinwirken; soziale Medien und die Handynutzung Jugendlicher folgen ohnehin nicht dem Pausenklingeln. Themen, Fragen und Probleme Jugendlicher wirken aus ihrem Alltag, online wie offline, in die Schule hinein und andersherum. Auch wenn Schulbetrieb und soziale Medien gegenwärtig wenig vereinbar scheinen, wäre es für Schule zunehmend wichtig, diese Herausforderung als Chance zu begreifen und das „Onlife“ der jungen Menschen im Schulalltag zum Gegenstand und - besser noch - zur Methode zu machen. So könnte sich schulbezogene Onlinebeteiligung entwickeln, die bestenfalls von Schülerinnen und Schülern selbst umgesetzt wird.

Eine entscheidende Frage blieb bei der Bewertung der Projekte noch unberührt. Wie weit kann - im pädagogischen Rahmen - die Beteiligung der Jugendlichen online reichen? Grundlegend sollte festgehalten werden, dass es sich vor allem um gesellschaftliche Teilhabe und soziale Partizipation handelt, wenn junge Menschen in den sozialen Medien ihrer Stimme Gehör verleihen. Politisch entschieden wird auf diesen Plattformen durch sie nichts. Sie können bestenfalls ihre Perspektiven zu gesellschaftspolitischen Debatten beitragen und diese mitgestalten, aber auch hier nur in einem beschränkten Maß, denn die in Bildungsprojekten erzielten Reichweiten sind überschaubar und Entscheidungsträgerinnen und Entscheidungsträger in Politik und Wirtschaft werden auf organischem Weg nicht erreicht. 
Junge Menschen in Bildungsprojekten, aber auch außerhalb davon, agieren online in anderen Filterblasen als Akteurinnen und Akteure in Politik oder Wirtschaft. Bestenfalls kann die Verschlagwortung ihrer Anliegen über Hashtags diese sichtbar bzw. findbar machen. In der Projektarbeit aber fehlen schlichtweg die Ressourcen, diese Distanzen zu überbrücken. Weder die finanziellen Mittel noch die Infrastruktur, wie Agenturen sie besitzen, um etwa professionelle Influencerinnen und Influencer zu promoten, stehen Jugendlichen oder Bildungsträgern zur Verfügung.

Jedoch stellt sich meines Erachtens nicht die Frage, ob es sich deswegen um weniger oder gar keine Beteiligung handelt. Onlinebeteiligung findet als politische Bildung nämlich auch dann statt, wenn sie nicht unmittelbar in politische Entscheidungen überführt wird, denn ausschlaggebend für den Bildungsprozess ist vielmehr die Entwicklung von Mündigkeit und Handlungskompetenz der Jugendlichen in den sozialen Medien. Wenn in Social-Media-Projekten die Themen und Anliegen der Jugendlichen bearbeitet und kontextualisiert werden und im Nachgang zu fundierten Haltungen führen, dann werden damit die Bedingungen von Onlinebeteiligung zuallererst geschaffen.

Geschieht diese Arbeit im öffentlichen Raum der sozialen Medien selbst, haben sie zudem ihre öffentliche Rolle erprobt und echte Verantwortung wahrgenommen. Ihre Medienkompetenz wurde in Anwendung erweitert und politische Bildung online ist dann kein simuliertes Handeln. Sie arbeitet explizit unter den politischen Bedingungen und gesellschaftlichen Verhältnissen des Mediums. So wird politische Bildung online selbst zu Beteiligung.

\section{Literatur}

Behrendt, R. (2019). Kriterien zur Challenge von how2influence. https://how2influence.de/ kriterien-fur-gute-social-medial-kanale/.

Brandes, C. (2016). \#NichtEuerErnst [YouTube Video]. https://www.youtube.com/watch?v= C4SS_Skg1XU.

Feierabend, S., Rathgeb, T., \& Reutter, T. (2018). Inhaltliche Aspekte der Internetnutzung. In Medienpädagogischer Forschungsverbund Südwest (mpfs) (Hrsg.), JIM-Studie 2018: Jugend, Information, Medien, Basisuntersuchung zum Medienumgang 12- bis 19-Jähriger (S. 31-37). Stuttgart: Medienpädagogischer Forschungsverbund Südwest (mpfs).

Floridis, L. (2015). Die 4. Revolution: Wie die Infosphäre unser Leben verändert. Berlin: Suhrkamp.

Steiner, P. (1993). On the Internet, nobody knows you're a dog [Cartoon]. https://en.wikipe dia.org/wiki/File:Internet_dog.jpg. 
Open Access Dieses Kapitel wird unter der Creative Commons Namensnennung 4.0 International Lizenz (http://creativecommons.org/licenses/by/4.0/deed.de) veröffentlicht, welche die Nutzung, Vervielfältigung, Bearbeitung, Verbreitung und Wiedergabe in jeglichem Medium und Format erlaubt, sofern Sie den/die ursprünglichen Autor(en) und die Quelle ordnungsgemäß nennen, einen Link zur Creative Commons Lizenz beifügen und angeben, ob Änderungen vorgenommen wurden.

Die in diesem Kapitel enthaltenen Bilder und sonstiges Drittmaterial unterliegen ebenfalls der genannten Creative Commons Lizenz, sofern sich aus der Abbildungslegende nichts anderes ergibt. Sofern das betreffende Material nicht unter der genannten Creative Commons Lizenz steht und die betreffende Handlung nicht nach gesetzlichen Vorschriften erlaubt ist, ist für die oben aufgeführten Weiterverwendungen des Materials die Einwilligung des jeweiligen Rechteinhabers einzuholen. 